DR. STEVE TURNER (Orcid ID : 0000-0001-8393-5060)

MS. EVELIEN R VAN MEEL (Orcid ID : 0000-0002-0826-9931)

PROF. ANKE-HILSE MAITLAND (Orcid ID : 0000-0002-0414-3442)

Article type : Original

\title{
Early life antibiotic use and the risk of asthma and asthma exacerbations in children
}

Fariba Ahmadizar ${ }^{1}$, Susanne J. H. Vijverberg ${ }^{1,2}$, Hubertus G. M. Arets $^{3}$, Anthonius de Boer ${ }^{1}$, Steve Turner ${ }^{4}$, Graham Devereux ${ }^{4}$, Ali Arabkhazaeli ${ }^{1}$, Patricia Soares ${ }^{5}$, Somnath Mukhopadhyay ${ }^{5,6}$, Johan Garssen $^{7,8}$, Colin N.A. Palmer ${ }^{9}$, Johan C. de Jongste ${ }^{10}$, Vincent WV Jaddoe ${ }^{11}$,Liesbeth Duijts ${ }^{10,11,12}$, Evelien R. van Meel ${ }^{10,11}$, Aletta D. Kraneveld ${ }^{7,13}$, Anke H. Maitland-van der Zee ${ }^{1,2^{*}}$

\section{Affiliation:}

1) Division of Pharmacoepidemiology and Clinical Pharmacology, Utrecht Institute for Pharmaceutical Sciences (UIPS), Faculty of science, Utrecht University, Utrecht, the Netherlands

2) Department of Respiratory Medicine, Academic Medical Centre, University of Amsterdam, Amsterdam, the Netherlands

3) Department of Pediatric Pulmonology and Allergology, Department of Pediatrics, University Medical Centre Utrecht, Utrecht, The Netherlands

4) Child Health, Royal Aberdeen Children's Hospital, Aberdeen, UK

5) Academic Department of Pediatrics, Brighton \& Sussex Medical School, Royal Alexandra Children's Hospital, Brighton, United Kingdom

6) Population Pharmacogenetics Group, Biomedical Research Institute, University of Dundee, Ninewells Hospital and Medical School, Dundee

7) Division of Pharmacology, Utrecht Institute for Pharmaceutical Sciences (UIPS), Faculty of science, Utrecht University, Utrecht, the Netherlands

8) Nutricia Research, Utrecht, the Netherlands

9) Population Pharmacogenetics Group, Biomedical Research Centre, University of Dundee, Dundee UK

10) Department of Pediatrics, Division of Respiratory Medicine and Allergology, Erasmus MC, University Medical Center, Rotterdam, The Netherlands

11) Department of Epidemiology, Erasmus MC, University Medical Center, Rotterdam, The Netherlands

12) Department of Pediatrics, Division of Neonatology, Erasmus MC, University Medical Center, Rotterdam, The Netherlands

13) Institute for Risk Assessment Sciences, Faculty of Veterinary Medicine, Utrecht University, Utrecht, the Netherlands

Running title: Antibiotic and risk of asthma/asthma exacerbations in children

*Corresponding authors:

Department of Respiratory Medicine, Academic Medical Centre, University of Amsterdam, Amsterdam, the Netherlands.

This article has been accepted for publication and undergone full peer review but has not been through the copyediting, typesetting, pagination and proofreading process, which may lead to differences between this version and the Version of Record. Please cite this article as doi: $10.1111 /$ pai.12725

This article is protected by copyright. All rights reserved. 
E-mail: a.h.maitland@amc.uva.nl

Post address: Department of Respiratory Disease, F5-259, P.O. Box 22660, The Netherlands

Tel: +31 (20) 5668137

Fax: (+31) (0)30-2539166

Word count: $\mathbf{2 , 6 6 7}$

Number of tables: 4

Number of figures: 2

Sources of support: No funding source had any role in the design and conduct of the study, the collection, management, analysis and interpretation of the data or the preparation, review or approval of the manuscript.

Ahmadizar. F, Vijverberg. S, Arets. H, de Boer. A, Turner. S, Devereux. G, Arabkhazaeli. A, Soares. P, Mukhopadhyay. S, Garssen. J, Palmer. C, de Jongste. J, Jaddoe. V, Duijts. L, van Meel. E, Kraneveld. A, Maitland-van der Zee. A

Early life antibiotic use and the risk of asthma and asthma exacerbations in children

\section{PAI journal}

\section{Abstract}

Background. The use of antibiotic therapy early in life might influence the risk of developing asthma. Studies assessing the influence of early life antibiotic use on the risk of asthma exacerbations are limited, and the results are inconsistent. Therefore, the aim of this study was to assess the association between use of antibiotic during the first three years of life and the risk of developing childhood asthma and the occurrence of asthma exacerbations.

Methods. Data from four large childhood cohorts were used; two population-based cohorts to study the risk of developing asthma: Generation $\mathrm{R}(\mathrm{n}=7,393$, the Netherlands) and SEATON $(\mathrm{n}=891$, Scotland, UK), and two asthma cohorts to assess the risk of asthma exacerbations: PACMAN ( $n=668$, the Netherlands) and BREATHE $(n=806$, Scotland, UK). Odds ratios (ORs) were derived from logistic regression analysis within each database followed by pooling the results using a fixed- or random-effect model.

Results. Antibiotic use in early life was associated with an increased risk of asthma in a meta-analysis of the Generation R and SEATON data (OR: 2.18, 95\% CI: 1.04-4.60; $I^{2}$ : $76.3 \%$ ). There was no association between antibiotic use in early life and risk of asthma exacerbations later in life in a meta-analysis of the PACMAN and BREATHE data (OR: $0.93,95 \%$ CI: $\left.0.65-1.32 ; \mathrm{I}^{2}: 0.0 \%\right)$. 
Conclusion. Children treated with antibiotic in the first three years of life are more likely to develop asthma, but there is no evidence that the exposure to antibiotic is associated with increased risk of asthma exacerbations.

Key words: Antibiotic, asthma, asthma exacerbations, early life, pediatrics.

Anke H. Maitland-van der Zee

Department of Respiratory Medicine, Academic Medical Centre, University of Amsterdam, Amsterdam, the Netherlands.

E-mail: a.h.maitland@amc.uva.nl

Post address: Department of Respiratory Disease, F5-259, P.O. Box 22660, The Netherlands

Tel: +31 (20) 5668137

\section{Introduction}

Several environmental and genetic factors have been identified as risk factors for asthma and asthma exacerbations (1-4), including early life risk factors such as antibiotic exposure. However, previous studies have shown conflicting findings whether antibiotic exposure in early life increases the risk of asthma development in children. Furthermore, the association between early life antibiotic use and severity of asthma is not well studied (5).

The aim of this study was to evaluate the effect of early life antibiotic use on the risk of asthma onset later in life in the general population. Moreover, in the pediatric population with asthma, the association between early life antibiotic use and risk of asthma exacerbations later in life was studied.

\section{Methods}

Study setting and population. To study the effect of antibiotic treatment early in life on asthma development, we used data from two population-based cohorts (Generation R (6) and SEATON $(7,8)$ ), and to assess the effect of early life antibiotic use on asthma exacerbations we used data from two pediatric asthma populations (PACMAN (9) and BREATHE (10, 11)). Generation $R$ is a Dutch prospective population-based cohort study $(n=7,393)$ of 
children from fetal life until young adulthood. Detailed information on the design and methods of this study has been described previously (6). SEATON is a Scottish birth cohort study (n=2,000). Between 1997 and 1999, healthy unselected pregnant women attending an antenatal clinic, at median 11 weeks gestation, were recruited. In this study, singletons born to the recruited women were followed up for 10 years $(7,8)$. PACMAN $(n=995)$, is a Dutch cohort of 4-12 year olds on asthma medication, selected through Dutch community pharmacies. In this cohort, children with at least two years of dispensed medication data available and at least three prescriptions for any asthma drug within the last two years and at least one prescription in the last six months were selected from pharmacies in the Netherlands (9). BREATHE is a Scottish cohort of children aged 3-19 years $(n=1,100)$ with physiciandiagnosed asthma recruited through primary or secondary clinics. The Community Health Index (CHI) coding was available for the BREATHE participants and data could be linked to several other databases e.g. community prescribing information and diagnosis of acute hospital admission $(10,11)$. Within the four cohorts questionnaire-based information was available on general health, allergic symptoms, asthma and respiratory symptoms, healthcare utilization for respiratory symptoms, environmental and socio demographic factors.

Exposure. Antibiotic use in the Generation R cohort (first three years) and in the SEATON cohort (first six months) was available from parental-reported data. In PACMAN and BREATHE, pharmacy data were available from the $1^{\text {st }}$ year of life, including detailed information on dispensed medication. In PACMAN, ATC codes (listed in Table S1) and in BREATHE, British National Formulary (BNF) codes (including all antibiotic in Chapter 5.1 of the BNF (12)) were used. Date of dispensing, duration, and amount dispensed and doses were extracted from these databases. In case of missing values, duration of antibiotic was calculated by assuming a course of 7 days. Depending on the available information in the four databases, antibiotic use was classified as 1) exposed vs. never exposed (in all four 
databases), 2) the timing of the first antibiotic use: $1^{\text {st }}$ year, the $2^{\text {nd }}$ year or the $3^{\text {rd }}$ year of life (in Generation R, PACMAN and BREATHE), 3) the number of prescriptions for antibiotic during the first three years (PACMAN and BREATHE), and 4) the total days of exposure to antibiotic during the first three years (PACMAN and BREATHE).

Outcomes. Two outcomes were defined in this study: 1) physician-diagnosed asthma, defined as a positive response to the following questions at age of 9-10 years in Generation $\mathrm{R}$ and at age of 10 years in SEATON "has your child ever suffered from asthma?" and "was this confirmed by a doctor?", and 2) asthma exacerbations, defined as asthma-related visits to an emergency department (ED) and/or the use of oral corticosteroids (OCS) in the past 12 months in PACMAN and asthma-related hospitalization and/or OCS use in the past 6 months in BREATHE(13). Since data on asthma control (measured with asthma control questionnaire (ACQ) were only available in PACMAN database, we were not able to study this outcome.

Statistical analysis. Children with missing values on exposure and outcomes were excluded from data analyses. The baseline characteristics of children with and without missing data were compared. Univariate and multivariable logistic regression analyses were used to estimate odds ratios (ORs) and 95\% confidence intervals (CIs) for the association between exposure and outcomes. Based on available variables in each database, the associations were adjusted for different confounders. In Generation R, the potential confounders were age, gender, and family history of asthma/allergy and in SEATON, gender, and family history of asthma. In PACMAN and BREATHE, age, gender and family history of asthma/allergy were included in the model. In PACMAN and BREATHE, we tested the association of number of courses and duration of antibiotic with asthma exacerbations. In subgroup analyses, the effect of age on this association was also tested in stratified analyses in three different age categories $>4$ and $\leq 6,>6$. and $\leq 13$ years in PACMAN and $>3$ and $\leq 6,>6$ and $\leq 13$ and $>13$. 
and $\leq 19$ years in BREATH. In all analyses children exposed to antibiotic were compared with unexposed children. The estimates for the association between antibiotic use and asthma in the Generation $\mathrm{R}$ and SEATON cohorts were meta-analyzed using a fixed-or randomeffect model. In the Generation $\mathrm{R}$ cohort, we only used the $1^{\text {st }}$ year of life of antibiotic exposure for better comparability with the SEATON cohort in which the exposure to antibiotic was only known for the first six months. A similar meta-analysis was performed for the association between antibiotic use and asthma exacerbations in PACMAN and BREATHE. All statistical analyses were conducted using statistical package $\mathrm{R}$ version 3.2.3 (14), SPSS version 21 and STATA 14/SE (15).

\section{Results}

In the population-based cohorts, 7,393 children in Generation $\mathrm{R}$ and 891 out of 2,000 children in SEATON had data available on both exposure to antibiotic and respiratory outcome. In Generation R, the rate of early life exposure to antibiotic ( $1^{\text {st }}$ year) was $55 \%$ while in SEATON, this rate (first 6 months) was 29\% (Table 1). Cumulative incidence rates of asthma in Generation $\mathrm{R}$ and SEATON were $10 \%$ and $8 \%$, respectively. In the asthma populations, 668 out of 995 (PACMAN) and 806 out of 1,100 (BREATHE) children had pharmacy data available starting from the $1^{\text {st }}$ year and were therefore included in this study. In these two databases, 78 and $84 \%$ of children had at least one antibiotic prescription during the first three years, respectively. The rate of children with at least one asthma exacerbation varied between $12 \%$ in PACMAN and $24 \%$ in BREATHE.

In the SEATON cohort, there were no statistically significant differences in baseline characteristics of children with and without missing data on early life antibiotic use except for family history of asthma (Table S2). When we compared the baseline characteristics of children who were lost to follow-up (unknown asthma diagnosis) with those included in the 
analyses, the results showed statistically significant difference in gender and family history of asthma between the two groups but not in exposure to antibiotics in early childhood (data not shown).

Early life antibiotic use and asthma susceptibility. In the two population-based cohorts, pooled results showed that children who used antibiotic during the $1^{\text {st }}$ year of life were statically significantly more likely to develop asthma (summary OR: $2.18,95 \%$ CI: $1.04-$ 4.60; $\mathrm{I}^{2}: 76.3 \%$ ) compared with those without antibiotic (Fig 1). In Generation R, antibiotic consumption in the $1^{\text {st }}$ year of life was associated with a higher risk of developing asthma (adjusted OR (aOR): $3.21,95 \%$ CI: $1.89-5.45)$ compared with antibiotic use in the $2^{\text {nd }}$ year of life (aOR: $2.25,95 \%$ CI: $1.18-4.30)$ or in the $3^{\text {rd }}$ year of life (aOR: $2.21,95 \%$ CI: $0.92-5.33$ ). In SEATON, antibiotic use during the first six months of life was not significantly associated with an increased risk of asthma (aOR: 1.50, 95\% CI: 0.91-2.46) (Table 2).

Early life antibiotic use and asthma exacerbations. In the two asthma populations, pooled results showed no associations between early life antibiotic use and asthma exacerbations (summary OR: $0.93,95 \%$ CI: $0.65-1.32 ; \mathrm{I}^{2}: 0.0 \%$ ) (Fig 2). No statistically significant differences between different timing of antibiotic use (first, second or third year of life) and the risk of asthma exacerbations were observed in PACMAN and BREATHE. In both studies, there were no significant associations between the number or duration of antibiotic courses and the risk of asthma exacerbations later in life (Table 3).

The results of age-stratified logistic regression analyses showed no statistically significant differences between different age categories in the two asthma populations. However, in both studies there seemed to be a trend towards a higher risk of asthma exacerbations in younger (4-6 years in PACMAN and 3-6 years in BREATHE) compared to older children (Table 4). 


\section{Discussion}

In this study, we showed children who had been exposed to antibiotic early in life had a higher risk of developing asthma. However, asthmatic children who were exposed to antibiotic early in life did not have a higher risk of asthma exacerbations.

In recent decades, a rise in the proportion of children treated with antibiotic in early childhood has been reported; the highest rate of antibiotic use (18.7\%) was shown in children aged $>1$ month to 18 years treated for a bacterial lower respiratory tract infection (16). Due to a high burden of infections in children in the first years of life the prescribing rate of antibiotic is high (in our study around 80\%). When antibiotic use in early life indeed increases the risk of asthma, it would be important to be more critical about the prescription of antibiotic. Studies have shown that antibiotics are often prescribed inappropriately $(17,18)$ for indications for which antibiotics are not necessary like common cold and sinusitis caused by viral infections.

Our findings regarding a higher risk of asthma in children exposed to antibiotic at an early age are in line with previous studies (19-23). In a very recent population-based birth cohort study of 143,000 children, a high risk of new-onset asthma was observed in children treated with antibiotic before the age of 12 months (incidence rate ratio: 1.51) (20). In the Generation $\mathrm{R}$ cohort, we showed that the strongest risk of asthma was in children exposed to antibiotic during the first year of life and the effect decreased if they were treated at an older age. Previous studies showed that the risk of asthma in children exposed to antibiotic in early life diminished with aging, and was no longer statistically significant after three years of age (24, 25). Our findings were also in line with the findings of a meta-analysis including 18 studies that reported a weak but statistically significant association between early life antibiotic exposure and the development of asthma (OR: 1.27, 95\% CI: 1.12-1.43) (19). 
A higher risk of asthma among children treated with antibiotic during early childhood could be explained in different ways. First, the immunomodulatory effect of antibiotic in which changes in the intestinal bacterial diversity during the first months of life influence the immune system, $\mathrm{T}$ helper and regulatory $\mathrm{T}$ cell (Tregs) balance. Evidence showed that modifiable factors such as gut microbiome and diet have a key role in the maturation of the neonatal immune system and thereby might also affect the risk of asthma and asthma exacerbations $(1,26,27)$. The healthy gut microbiome is highly diverse and can be disturbed by environmental factors e.g. antibiotic therapy.

Another explanation for the higher risk could be confounding by indication. It is possible that children who are in need of antibiotic early in life, have already a Th1/Th2 imbalance caused by the infections treated with the antibiotic and that this makes them more susceptible for asthma later in life. In the Generation $\mathrm{R}$ and SEATON cohorts, we cannot rule out that respiratory symptoms in early life treated with antibiotic might have been the first signs of asthma.

Prevalence rate of asthma exacerbations varied widely between PACMAN (12\%) and BREATHE (24\%) studies; children in the BREATHE study have approximately twice the risk of asthma exacerbations. Importantly, children in the PACMAN study were recruited through community pharmacies based on their regular use of medication for asthma treatment, while participants of the BREATHE were recruited through primary and secondary asthma clinics. Therefore, the children included in BREATHE might reflect a more severe asthma population who more often suffer from asthma exacerbations. Cultural differences in OCS prescriptions in different countries might also explain part of the discrepant results. However, the results of the association between antibiotic and asthma exacerbations were very similar in PACMAN and BREATHE; even though the point estimates were in opposite directions the $95 \%$ CIs largely overlapped. A previous study reported a statistically 
significant positive association between antibiotic use and asthma exacerbations shortly after treatment with antibiotic (before the age two years), and not at older age (5). The latter finding is in line with the findings in our study, however, we did not have data to study asthma exacerbations before the age of two years.

There are some potential limitations in this study that should be acknowledged. Importantly, our study was limited by the high rate of missing data in the three studies including SEATON (56\%), PACMAN (33\%) and BREATHE (27\%). When assessing the baseline characteristics of the children with and without missing data, in SEATON cohort the results showed no statistically significant differences in asthma development between children with and without missing data on antibiotic use in early childhood. Therefore, our study was not prone to selection bias. However, the results of analyses in the two asthma populations showed statistically significant differences in asthma exacerbations between the two groups in which those who had missing data on early life medications had remarkably less asthma exacerbations compared with those with available data in PACMAN (6.1\% vs $12.3 \%$, $\mathrm{p}=0.003)$ and in BREATHE (11.6\% vs $24.3 \%, \mathrm{p}=0.01)$. Therefore, this might have led to an overrepresentation of children with more severe asthma in the analyses.

Another important limitation in this study was the heterogeneity in study designs. For example in Generation R antibiotic use in the first three years of life were assessed, while the first six months of life were assessed in SEATON. Different time periods for antibiotics exposure (and therefore different age at time of antibiotic consumption) might have led to a stronger association in Generation R compared with SEATON study. In the asthma studies antibiotic exposure could be defined based on pharmacy data, while these data were not available in the population based studies. In these studies, the use of antibiotic was parental reported, this definition might be more prone to recall bias. 
The definition of asthma diagnosis in both SEATON and Generation $\mathrm{R}$ was based on a parental questionnaire. This might have led to misclassification of asthmatic children especially when the diagnosis was obtained at a young age or an under-reporting of asthma cases. However, we previously showed that there is a high agreement (89\%) between physician-diagnosis asthma and parental-reported asthma in the PACMAN study (28).

Heterogeneity in confounders involved in the adjusted models is another limitation. The associations in different populations were adjusted for different confounders based on available variables in each study. Lack of consistent adjustment in the present study might have influenced the associations; the possibility remains that some factors which were not measured in every population caused confounding.

The present study was also limited by the lack of availability of potential confounders such as genetic factors, socioeconomic status and infections/siblings. In none of the databases, we had information about the indications for the prescribing of antibiotic.

In summary, our findings show a higher risk of asthma in children who are treated with antibiotic early in life, but no association of early life antibiotic use and asthma exacerbations later in asthmatic children. We propose that further research is needed to explore the effects of antibiotic on the immune system and gut microbiome in the pathogenesis of asthma.

Acknowledgements The Generation R Study is conducted by the Erasmus Medical Centre in close collaboration with the School of Law and the Faculty of Social Sciences at the Erasmus University, Rotterdam, the Municipal Health Service, Rotterdam area, and the Stichting Trombosedienst and Artsenlaboratorium Rijnmond (Star-MDC), Rotterdam. We gratefully acknowledge the contribution of children and parents, general practitioners, hospitals, midwives and pharmacies in Rotterdam. 
We also wish to acknowledge the support of the Health Informatics Centre, University of

Dundee for managing and supplying the anonymized data.

\section{References:}

1. O'Dwyer DN, Dickson RP, Moore BB. The Lung Microbiome, Immunity, and the Pathogenesis of Chronic Lung Disease. J Immunol 2016: 196: 4839-4847.

2. Riiser A. The human microbiome, asthma, and allergy. Allergy Asthma Clin Immunol 2015: 11: 35-015-0102-0. eCollection 2015.

3. Abrahamsson TR, Jakobsson HE, Andersson AF, Bjorksten B, Engstrand L, Jenmalm MC. Low gut microbiota diversity in early infancy precedes asthma at school age. Clin Exp Allergy 2014: 44: 842-850.

4. Bonnelykke $\mathrm{K}$, Ober C. Leveraging gene-environment interactions and endotypes for asthma gene discovery. J Allergy Clin Immunol 2016: 137: 667-679.

5. Semic-Jusufagic A, Belgrave D, Pickles A, et al. Assessing the association of early life antibiotic prescription with asthma exacerbations, impaired antiviral immunity, and genetic variants in 17q21: a population-based birth cohort study. Lancet Respir Med 2014: 2: 621630.

6. Jaddoe VW, van Duijn CM, Franco OH, et al. The Generation R Study: design and cohort update 2012. Eur J Epidemiol 2012: 27: 739-756.

7. Devereux G, Turner SW, Craig LC, et al. Low maternal vitamin E intake during pregnancy is associated with asthma in 5-year-old children. Am J Respir Crit Care Med 2006: 174: 499507.

8. Martindale S, McNeill G, Devereux G, Campbell D, Russell G, Seaton A. Antioxidant intake in pregnancy in relation to wheeze and eczema in the first two years of life. Am $\mathbf{J}$ Respir Crit Care Med 2005: 171: 121-128.

9. Koster ES, Raaijmakers JA, Koppelman GH, et al. Pharmacogenetics of anti-inflammatory treatment in children with asthma: rationale and design of the PACMAN cohort. Pharmacogenomics 2009: 10: 1351-1361.

10. Tavendale R, Macgregor DF, Mukhopadhyay S, Palmer CN. A polymorphism controlling ORMDL3 expression is associated with asthma that is poorly controlled by current medications. J Allergy Clin Immunol 2008: 121: 860-863.

11. Palmer CN, Lipworth BJ, Lee S, Ismail T, Macgregor DF, Mukhopadhyay S. Arginine-16 beta2 adrenoceptor genotype predisposes to exacerbations in young asthmatics taking regular salmeterol. Thorax 2006: 61: 940-944.

12. Anonymous BNF for Children 2012. British Medical Association. The Royal Pharmaceutical Society of Great Britain and Royal College of Pediatrics and Child Health and Pediatric Neonatal Group, 2012.

13. Wu AC, Tantisira K, Li L, et al. Predictors of symptoms are different from predictors of severe exacerbations from asthma in children. Chest 2011: 140: 100-107.

14. Anonymous $\mathrm{R}$ development core team. R: A language and environment for statistical computing.

Found. stat. comput. (2015).

15. Anonymous StataCorp. 2015. Stata Statistical Software: Release 14. College Station, TX: StataCorp LP.

16. Versporten A, Bielicki J, Drapier N, Sharland M, Goossens H, ARPEC project group. The Worldwide Antibiotic Resistance and Prescribing in European Children (ARPEC) point 
prevalence survey: developing hospital-quality indicators of antibiotic prescribing for children. J Antimicrob Chemother 2016: 71: 1106-1117.

17. Smith SM, Fahey T, Smucny J, Becker LA. Antibiotics for acute bronchitis. Cochrane Database Syst Rev 2014: 3: CD000245.

18. Spinks A, Glasziou PP, Del Mar CB. Antibiotics for sore throat. Cochrane Database Syst Rev 2013: 11: CD000023.

19. Penders J, Kummeling I, Thijs C. Infant antibiotic use and wheeze and asthma risk: a systematic review and meta-analysis. Eur Respir J 2011: 38: 295-302.

20. Pitter G, Ludvigsson JF, Romor P, et al. Antibiotic exposure in the first year of life and later treated asthma, a population based birth cohort study of 143,000 children. Eur J Epidemiol 2016: 31: 85-94.

21. Ortqvist AK, Lundholm C, Kieler $\mathrm{H}$, et al. Antibiotics in fetal and early life and subsequent childhood asthma: nationwide population based study with sibling analysis. BmjBritish Medical Journal 2014: 349: g6979.

22. Murk W, Risnes KR, Bracken MB. Prenatal or early-life exposure to antibiotics and risk of childhood asthma: a systematic review. Pediatrics 2011: 127: 1125-1138.

23. Ong MS, Umetsu DT, Mandl KD. Consequences of antibiotics and infections in infancy: bugs, drugs, and wheezing. Ann Allergy Asthma Immunol 2014: 112: 441-445.e1.

24. Ong MS, Umetsu DT, Mandl KD. Consequences of antibiotics and infections in infancy: bugs, drugs, and wheezing. Ann Allergy Asthma Immunol 2014: 112: 441-445.e1.

25. Ortqvist AK, Lundholm $\mathrm{C}$, Kieler $\mathrm{H}$, et al. Antibiotics in fetal and early life and subsequent childhood asthma: nationwide population based study with sibling analysis. BMJ 2014: 349: g6979.

26. Gollwitzer ES, Marsland BJ. Impact of Early-Life Exposures on Immune Maturation and Susceptibility to Disease. Trends Immunol 2015: 36: 684-696.

27. McFadden JP, Thyssen JP, Basketter DA, Puangpet P, Kimber I. T helper cell 2 immune skewing in pregnancy/early life: chemical exposure and the development of atopic disease and allergy. Br J Dermatol 2015: 172: 584-591.

28. Anonymous Pieters LE, Vijverberg SJH, Raaijmakers JAM, Van der Ent CK, Maitlandvan der Zee AH. Astmadiagnose bij kinderen. Huisarts Wet 2014;57(9):446-51. 
Table 1. Characteristics of the Generation R, SEATON, PACMAN and BREATHE studies

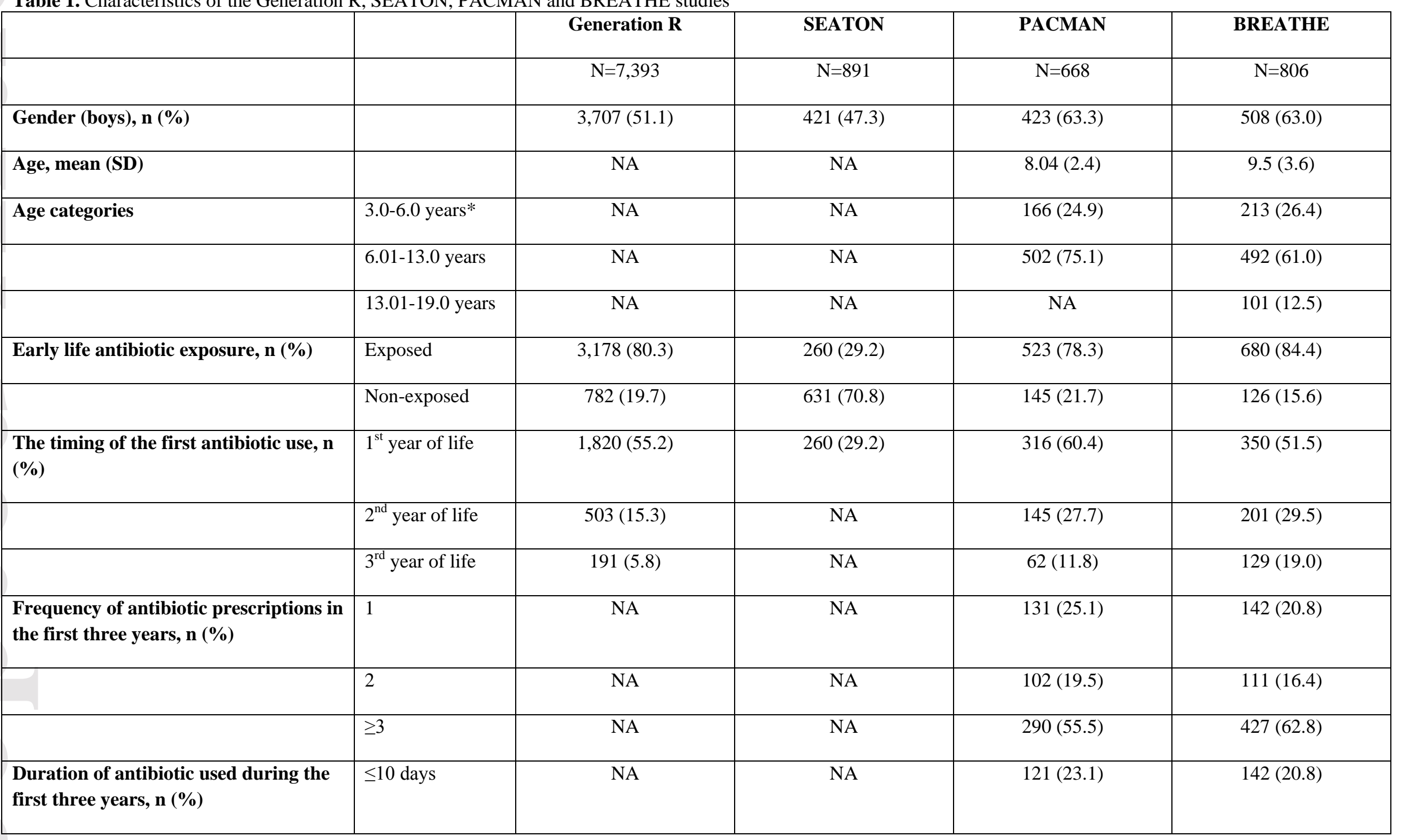

This article is protected by copyright. All rights reserved. 


\begin{tabular}{|c|c|c|c|c|c|}
\hline & 11-30 days & NA & NA & $225(43.0)$ & $270(39.7)$ \\
\hline & $>31$ days & NA & NA & $176(33.7)$ & $268(39.4)$ \\
\hline Asthma diagnosis, n (\%) & & $448(9.5)$ & $74(8.3)$ & NA & NA \\
\hline Exacerbations**, n (\%) & & NA & NA & $82(12.3)$ & $196(24.3)$ \\
\hline Family history of asthma $^{* * * *}, \mathrm{n}(\%)$ & & $664(16.7)$ & $218(24.5)$ & $305(47.5)$ & $315(39.8)$ \\
\hline $\begin{array}{l}\text { Family history of allergy/atopy }{ }^{* * * *}, \mathbf{n} \\
(\%)\end{array}$ & & $2,739(60.5)$ & NA & $524(81.0)$ & $351(44.4)$ \\
\hline
\end{tabular}

Abbreviations: SD, standard deviation; NA, not available

Early life antibiotic use in Generation R (first three years) and SEATON (first six months) is based on parental-reported data.

Prescribing data for the $1^{\text {st }}$ years of life was available in PACMAN and BREATHE.

*This age category is 4-6 years in the PACMAN study.

**Asthma exacerbations defined as at least one course oral corticosteroids use and/or at least one emergency department visits/hospitalizations due to asthma

${ }^{* * * *}$ At least one asthmatic parent

At least one allergic/atopic parent 


\section{Study; Odds ratio (95\% confidence interval)}

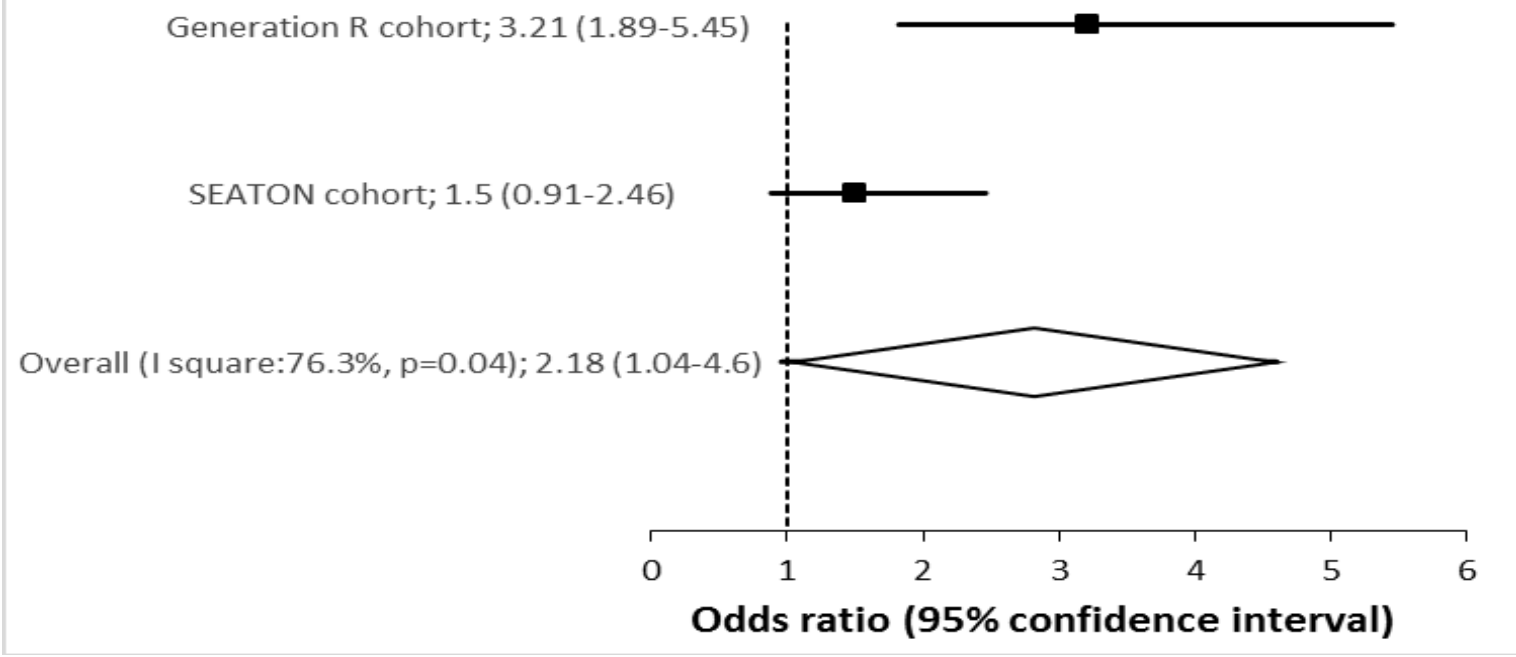

Fig 1. Pooled odds ratio of the association between early life exposure to antibiotic and asthma development, using random-effect model 
Table 2. Associations between early life exposure to antibiotic and asthma development

\begin{tabular}{|c|c|c|}
\hline & Crude OR (95\% CI) & *Adjusted OR (95\% CI) \\
\hline \multicolumn{3}{|l|}{ Generation $\mathbf{R}$} \\
\hline \multicolumn{3}{|l|}{$\begin{array}{l}\text { Ever exposed vs. never exposed to antibiotic, } \\
n=7,393\end{array}$} \\
\hline Ever exposed, $n=3,178$ & $3.29(2.17-5.00)$ & $2.84(1.70-4.75)$ \\
\hline Never exposed, $n=782$ & \multicolumn{2}{|c|}{ Reference } \\
\hline $1^{\text {st }}$ year of life antibiotic use, $n=1,820$ & $3.68(2.40-5.65)$ & $3.21(1.89-5.45)$ \\
\hline $2^{\text {nd }}$ year of life antibiotic use, $n=503$ & $2.70(1.61-4.54)$ & $2.25(1.18-4.30)$ \\
\hline $3^{\text {rd }}$ year of life antibiotic use, $n=191$ & $2.63(1.35-5.11)$ & $2.21(0.92-5.33)$ \\
\hline \multicolumn{3}{|l|}{ SEATON } \\
\hline \multicolumn{3}{|l|}{$\begin{array}{l}\text { Ever exposed vs. never exposed to antibiotic, } \\
n=891\end{array}$} \\
\hline Ever exposed, $n=260$ & $1.54(0.94-2.52)$ & $1.50(0.91-2.46)$ \\
\hline Never exposed, $n=631$ & \multicolumn{2}{|c|}{ Reference } \\
\hline
\end{tabular}

Abbreviations: OR, odds ratio; CI, confidence interval

Reference is never exposed group in all analyses.

Bold numbers: statistically significant associations.

*Adjusted for age, gender, and family history of asthma/allergy in Generation R, and for gender, and family history of asthma in SEATON, using multivariable logistic regression analysis.

This article is protected by copyright. All rights reserved. 
Table 3. Associations between early life exposure to antibiotic and asthma exacerbations

\begin{tabular}{|c|c|c|c|}
\hline & & Crude OR (95\% CI) & *Adjusted OR (95\% CI) \\
\hline \multicolumn{4}{|l|}{ PACMAN } \\
\hline \multicolumn{4}{|l|}{$\begin{array}{l}\text { Ever exposed vs. never exposed to } \\
\text { antibiotic, } n=668\end{array}$} \\
\hline Ever exposed, $n=523$ & & $1.16(0.65-2.08)$ & $1.09(0.60-1.96)$ \\
\hline Never exposed, $n=145$ & & \multicolumn{2}{|c|}{ Reference } \\
\hline \multirow{3}{*}{$\begin{array}{l}\text { Frequency of antibiotic } \\
\text { use }^{* *}, \mathrm{n}=668\end{array}$} & 1 antibiotic, $\mathrm{n}=131$ & $0.74(0.33-1.66)$ & $0.68(0.30-1.56)$ \\
\hline & 2 antibiotics, $\mathrm{n}=102$ & $1.39(0.65-2.96)$ & $1.19(0.54-2.61)$ \\
\hline & $\geq 3$ antibiotics, $\mathrm{n}=290$ & $1.29(0.70-2.39)$ & $1.25(0.67-2.34)$ \\
\hline \multirow{3}{*}{$\begin{array}{l}\text { Cumulative duration of antibiotic } \\
\text { use, } n=667\end{array}$} & $\leq 10$ days $n=121$ & $0.81(0.36-1.81)$ & $0.67(0.28-1.58)$ \\
\hline & $11-30$ days $n=225$ & $1.01(0.52-1.96)$ & $0.95(0.48-1.87)$ \\
\hline & $\geq 30$ days $n=176$ & $1.66(0.86-3.18)$ & $1.61(0.83-3.13)$ \\
\hline \multicolumn{4}{|l|}{ BREATHE } \\
\hline \multicolumn{4}{|l|}{$\begin{array}{l}\text { Ever exposed vs. never exposed to } \\
\text { antibiotic, } n=806\end{array}$} \\
\hline Ever exposed, $n=680$ & & $0.81(0.53-1.24)$ & $0.85(0.55-1.32)$ \\
\hline Never exposed, $n=126$ & & \multicolumn{2}{|c|}{ Reference } \\
\hline \multirow{3}{*}{$\begin{array}{l}\text { Frequency of antibiotic use }{ }^{* *} \text {, } \\
\mathbf{n}=680\end{array}$} & 1 antibiotic, $\mathrm{n}=142$ & $0.88(0.51-1.52)$ & $0.99(0.57-1.71)$ \\
\hline & 2 antibiotics, $\mathrm{n}=111$ & $0.72(0.40-1.30)$ & $0.78(0.42-1.44)$ \\
\hline & $\geq 3$ antibiotics, $\mathrm{n}=427$ & $0.81(0.51-1.26)$ & $0.82(0.52-1.31)$ \\
\hline \multirow{3}{*}{$\begin{array}{l}\text { Cumulative duration of antibiotic } \\
\text { use, } n=680\end{array}$} & $\leq 10$ days, $n=142$ & $0.88(0.51-1.52)$ & $0.99(0.57-1.71)$ \\
\hline & $11-30$ days, $n=270$ & $0.82(0.51-1.33)$ & $0.87(0.53-1.43)$ \\
\hline & $\geq 30$ days, $n=268$ & $0.75(0.46-1.22)$ & $0.76(0.46-1.25)$ \\
\hline
\end{tabular}

Abbreviations: OR, odds ratio; CI, confidence interval

Reference is never exposed group in all analyses.

*Adjusted for age, gender and family history of asthma/allergy, using multivariable logistic regression analysis.

** Number of courses of antibiotic

Asthma exacerbations defined as at least one course oral corticosteroids use and/or at least one emergency department visits/hospitalizations due to asthma;

This article is protected by copyright. All rights reserved. 


\section{Exposed vs. never exposed to antibiotics}

PACMAN study; 1.09 (0.6-1.96)

BREATHE study; $0.85(0.55-1.32)$

Overall (I square:0.0\%, p=0.68); 0.93 (0.65-1.32)

\section{1 course of antibiotics vs. never antibiotics \\ PACMAN study; 0.68 (0.3-1.56) \\ BREATHE study; 0.99 (0.57-1.71) \\ Overall (I square:0.0\%, p=0.59); 0.88 (0.56-1.39)}

\section{2 courses of antibiotics vs. never antibiotics}

PACMAN study; $1.19(0.54-2.61)$

BREATHE study; $0.78(0.42-1.44)$

Overall (I square:0.0\%, $\mathrm{p}=0.72) ; 0.92(0.56-1.49)$

$\geq 3$ courses of antibiotics vs. never antibiotics

PACMAN study; 1.25 (0.67-2.34)

BREATHE study; 0.82 (0.52-1.31)

Overall (I square:11.5\%, p=0.84); 0.96 (0.64-1.43)

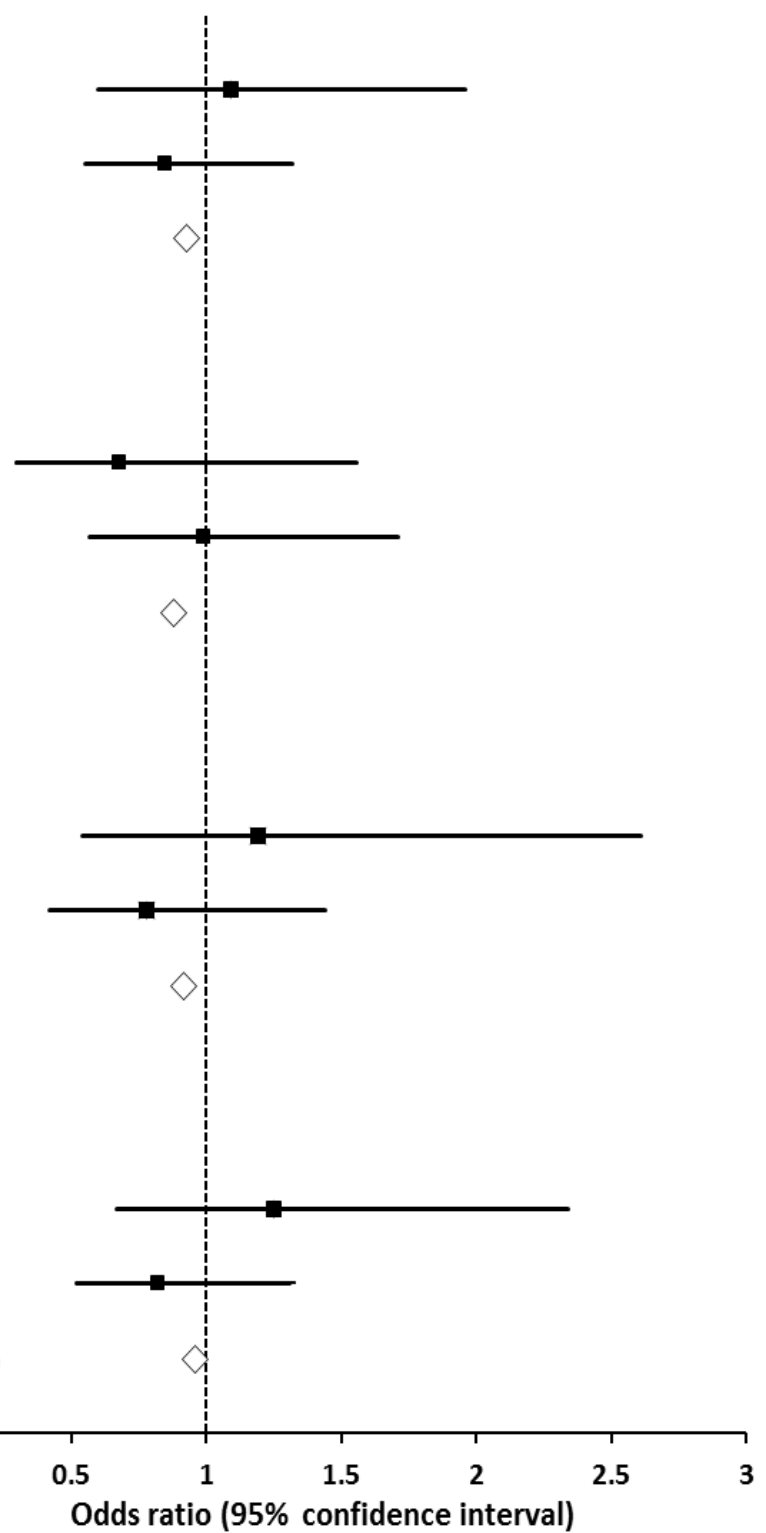

Fig 2. Pooled odds ratio of the associations between early life exposure to antibiotics and asthma exacerbations, using fixed-effect model

This article is protected by copyright. All rights reserved. 
Table 4: Associations between early life antibiotic use and asthma exacerbations, stratified by age

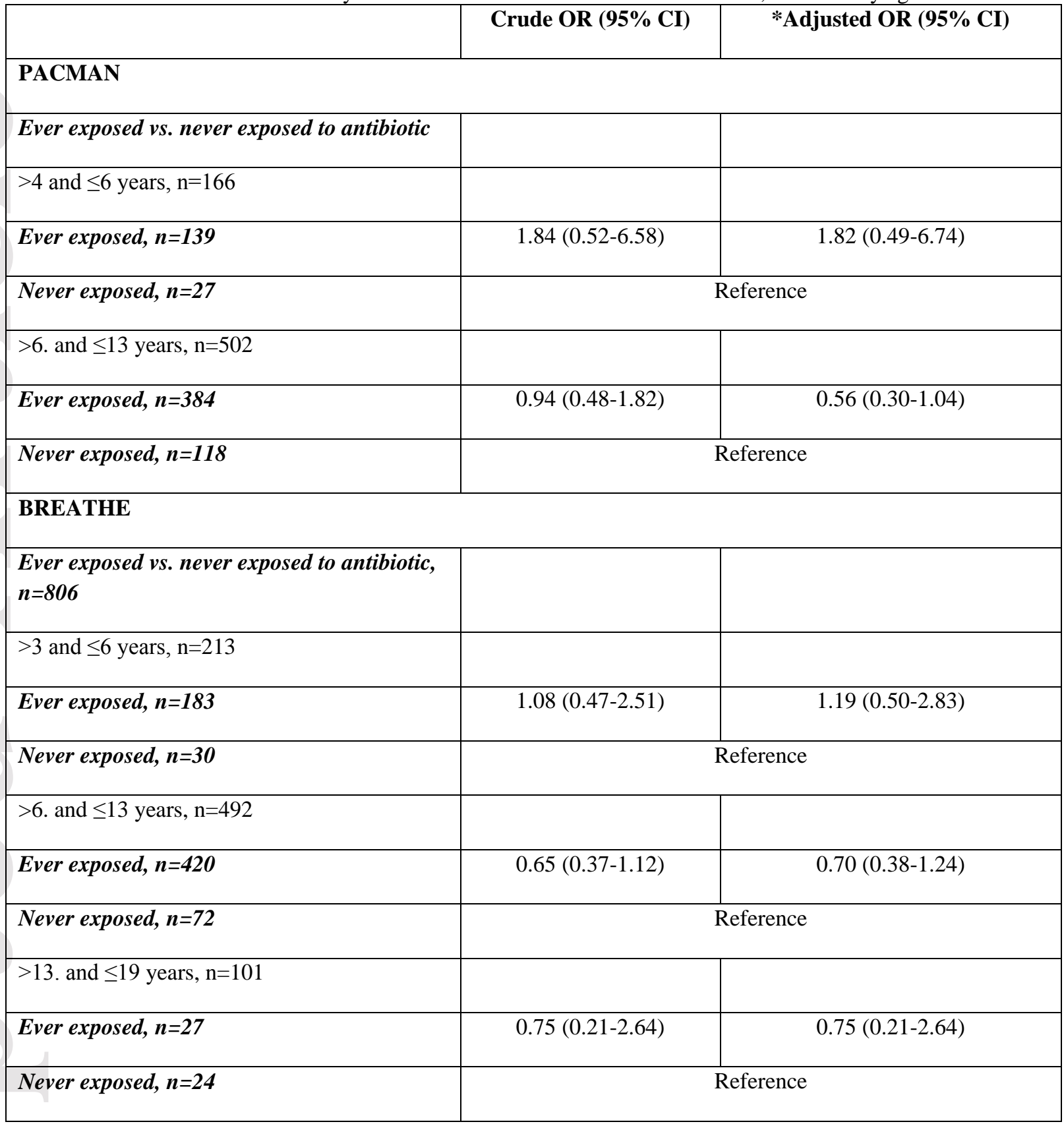

Abbreviations: OR, odds ratio; CI, confidence interval

*Adjusted for gender and family history of asthma/allergy;

Asthma exacerbations defined as at least one course oral corticosteroids use and/or at least one emergency department visits/hospitalizations due to asthma.

This article is protected by copyright. All rights reserved. 